\title{
Failure to Detect Metric Measurement Non-Invariance: How Manifest Residual Variances, Indicator Communalities, and Sample Size affect the $\chi^{2}$-Test Statistic
}

\author{
Eric Klopp* \\ Department of Education \\ Saarland University
}

\author{
Stefan Klößner \\ Statistics and Econometrics \\ Saarland University
}

December 9, 2020

\begin{abstract}
We investigate the effects of manifest residual variances, indicator communalities, and sample size on the $\chi^{2}$-test statistic of the metric measurement invariance model when the model is misspecified, i.e., there is at least one population loading that violates metric measurement invariance. First, we demonstrate the choice of the scaling method does not affect the model's $\chi^{2}$-test statistic. Afterward, we demonstrate that the $\chi^{2}$-test statistic relates inversely to manifest residual variances, whereas sample size and $\chi^{2}$-test statistic show a positive relation. Moreover, we consider indicator communality as a key factor for the size of the $\chi^{2}$-test statistic. In this context, we introduce the concept of signal-to-noise ratio as a tool for studying the effects of manifest residual variance and indicator communality and demonstrate its use with the example. Finally, we discuss the limitations and the practical implications for the analysis of metric measurement invariance models.
\end{abstract}

Keywords: metric measurement invariance, indicator communality, manifest residual variance, signal-to-noise ratio, Monte Carlo simulation

${ }^{*}$ Corresponding author: Eric Klopp, Saarland University, Department of Education, Bldg. A 4.2, 66123 Saarbrücken, Germany, email: e.klopp@mx.uni-saarland.de. 


\section{Introduction}

Measurement invariance analysis has become a widely used tool in many areas of applied research, e.g., psychology, sociology, and organizational research (Davidov, Muthen, \& Schmidt, 2018; Greiff \& Scherer, 2018; van de Schoot, Schmidt, \& de Beuckelaer, 2015), and received a lot of attention in methodological research (e.g., Millsap, 2011). The goal of measurement invariance (MI) analysis is to investigate whether measurement properties of a latent variable's indicators are the same across two or more groups. ${ }^{1}$ Usually, there are several levels of MI that are successively investigated (e.g., Brown, 2015, pp. 242-243). The lowest level is configural MI. At this level, the factor structure is the same in all groups. At the next level, metric MI, not only the factor structure is the same in all groups but the loadings of all indicators are invariant across groups. Thus, metric invariance entails configural invariance. A third level is scalar MI, which means that the factor structure, the loadings, and the intercepts are invariant across groups.

MI analysis draws on testing nested models by means of $\chi^{2}$-difference tests. ${ }^{2}$ For instance, once it is established that configural MI holds, i.e., a multi-group model with equal structure in each group fits the data well, equality constraints are placed on all loadings so that they are invariant across all groups. This model with equality constraints on the loadings is called the metric MI model. In case this metric MI model fits the data well, too, a $\chi^{2}$-difference test is conducted. In case this $\chi^{2}$-difference test is significant, the hypothesis of equal loadings is discarded because the metric invariance constraints deteriorate model fit significantly. When the $\chi^{2}$-difference test is not significant, the metric MI model is retained. A detailed description of this process can be found in, e.g, Jiang, Mai, and Yuan (2017). In this contribution, we only consider the metric MI model, so we do not elaborate on further MI levels. Moreover, we do not consider the $\chi^{2}$-difference test to compare the configural and metric MI model. The focus of this contribution is on the metric MI model and its $\chi^{2}$-test statistic.

As the metric MI model entails latent variables, it is a well known fact that scaling restrictions must be placed, otherwise the models would not be identified and could not be estimated. Importantly, scaling method enforce restrictions on estimated parameters (Klopp \& Klößner, in press). The Fixed Marker (FM) scaling (cf. Kline, 2016, pp. 199-200) is the most commonly applied scaling method and is the de facto standard in most structural equation software. The FM method consists in restricting one of the estimated loadings of any

\footnotetext{
${ }^{1}$ In this contribution, we will only consider a two group case and do not explicitly refer to longitudinal MI analysis, but the argumentation applies straightforwardly to settings with more than two groups and longitudinal models.

${ }^{2}$ Besides the $\chi^{2}$-difference test, there are other methods to compare the configural and metric MI model by means of other goodness-of-fit indices, e.g., Chen (2007), Cheung and Rensvold (2002), and Meade, Johnson, and Braddy (2008), which we will not consider in this contribution.
} 
latent variable to 1 , the respective manifest variable is called the referent indicator. Usually, it is suggested that the indicator that best represents the latent variable should be the referent indicator. In the metric MI model, usually one loading per latent variable and group is fixed to 1 and also the estimated loadings are equated across groups. In the context of metric MI analysis, the use of the FM method has been critically discussed. According to Brown (2015, p. 271), Cheung and Rensvold (1999), and Millsap (2011, pp. 85-86), there will be difficulties in multiple group settings regarding the selection of a referent indicator. Firstly, non-invariance of the referent indicator may not be detected, because it is fixed to 1 in every group. Thus, there is the implicit assumption that the referent indicator is invariant across groups. Secondly, this implicit invariance assumption may cause following tests of partial MI (i.e., not all estimated loadings are equated over the groups) to be distorted, because they are influenced by the choice of a non-invariant referent indicator and differences in loadings may reflect artifacts from this choice. Millsap (2011, pp. 85-86) exemplified that using a referent indicator which is non-invariant across groups may yield wrong conclusions when partial MI is investigated. Recently, Raykov, Marcoulides, Harrison, and Zhang (2020) raised concerns about the use of FM scaling when the referent indicator is non-invariant across groups even in a full metric MI analysis. These authors presented an example of a one factor model with 12 manifest indicators in two groups. The population model entails one indicator that is noninvariant across groups. In a Monte Carlo simulation they showed that on average, the metric model's $\chi^{2}$-test statistic is non-significant and thus, the model would fail, on average, to detect the non-invariance and metric MI would erroneously be accepted. However, this result is in contrast to Johnson, Meade, and DuVernet (2009), who reported that in a simulation study the choice of non-invariant referent indicators does not affect tests of metric MI.

Before we describe two further scaling methods, we want to remark that the FM scaling method has different variants in a given model. For instance, when there are 12 indicators, then there are 12 possible indicators whose estimated loading can be restricted to 1 . In this paper, if the first indicator is the referent indicator, then we use the abbreviation FM1. If the second indicator is the referent indicator, we write FM2, and so on.

Besides the FM scaling method, there are two other methods to scale latent variables in multiple group settings with metric MI restrictions (cf. Kline, 2016, pp. 199-200). In the Fixed Factor (FF) method, the variances of the latent variables are restricted to 1 . In the context of the metric MI model, the variant of the FF method is called the Reference Group (RG) method and the estimated latent variances is fixed to 1 in the reference group and freely estimated in the other groups while having the estimated loadings equated over the groups (cf. Kline, 2016, p. 393, Lee, Little, \& Preacher, 2011, p. 60). There is also the effects coding (EC, Little, Slegers, \& Card, 2006) method that works with constraints on the estimated 
loadings. These are constrained so that the average of the estimated factor loadings equals 1. In the metric MI model, this restriction applies in a first group and the estimated loadings are set equal across the other groups.

Obviously, in these scaling methods there are no assumptions about the invariance of a referent indicator. Indeed, van de Schoot, Lugtig, and Hox (2012) recommend using RG method when the goal is to scrutinize metric MI. Due to the controversial findings presented by Raykov et al. (2020) and Johnson et al. (2009) and the availability of two other scaling methods in multiple group settings, the question arises if there are effects on the $\chi^{2}$-test statistic of the metric MI model resulting from the choice of a particular scaling method or the choice of a particular referent indicator when the FM method is used. Moreover, drawing on the results of Johnson et al. (2009), the question arises why the findings presented in Raykov et al. (2020) emerged.

In this contribution, we firstly demonstrate by means of Monte Carlo simulation that the choice of a particular scaling method, or the choice of a particular referent indicator when using the FM method, does not affect the $\chi^{2}$-test statistic of the metric MI model, i.e., they all yield the same $\chi^{2}$-test statistic. Afterwards, using some results about the properties of structural equation models provided in Klopp and Klößner (in press), we demonstrate that all scaling methods in the metric MI model are tantamount, i.e., yield the same $\chi^{2}$-test statistic. Additionally, we demonstrate that the estimates of a model estimated under any given scaling method can be calculated using the estimates obtained under any other scaling method, i.e., we demonstrate that the parameters are convertible without the need to re-estimate the model. For this part, we draw on the example provided in Raykov et al. (2020). By using this example, we follow the call from the authors to do further research into the topic and generalize these authors findings (Raykov et al., 2020, p. 654). First, we demonstrate and explain why in a metric invariance setting all possible scaling method provide the same the $\chi^{2}$ test statistic. To this end, we use Monte Carlo simulation and theoretical reasoning regarding the scaling methods. Afterward, we scrutinize why in this example the $\chi^{2}$-test statistic fails to detect the deviation from metric MI. Again by means of Monte Carlo demonstrations, we demonstrate that the metric MI model's $\chi^{2}$-test statistic depends on the manifest residual variances and sample size. if the model is misspecified. Whereas the relation between sample size and $\chi^{2}$-test statistic is well-known, the effects of manifest residual variances have only been considered marginally (Heene, Hilbert, Draxler, Ziegler, \& Bühner, 2011). We introduce the concept of signal-to-noise ratio as a joint measure of manifest residual variance and indicator communality. We demonstrate the effects of the signal-to-noise ratio and sample size on the $\chi^{2}$-test statistic of the metric MI model. All simulation in this paper were done using Mplus 
(Muthén \& Muthén, 1998-2019) in version 8.3 with 10, 000 replications and the ML estimator. The Mplus scripts and the respective output can be found on OSF, see https://osf.io/pm3xk/.

\section{Scaling method and the $\chi^{2}$-test statistic}

In Raykov et al. (2020), the authors provide an example that consists of a population model with two groups and one latent variable. In detail, they use the following factor analytic model

$$
\boldsymbol{y}_{g}=\boldsymbol{\alpha}_{g}+\boldsymbol{\Lambda}_{g} \cdot \boldsymbol{\eta}_{g}+\boldsymbol{\delta}_{g}
$$

with $g \in\{1,2\}$ indexing the group, $g=1$ indicating the reference group and $g=2$ indicating the focal group (see Holland \& Thayer, 1988). Let $p$ be the number of manifest variable and $n$ the number of latent variables, then $\boldsymbol{y}_{g}$ is a $p$-dimensional vector of manifest variables, $\boldsymbol{\alpha}_{g}$ is a $p$-dimensional vector of latent intercepts, $\boldsymbol{\Lambda}_{g}$ is a $p \times n$ loading matrix, $\boldsymbol{\delta}_{g}$ is a zero-mean vector of error terms, whose variances are called manifest residual variances. Under standard assumptions (e.g., Bollen, 1989), this yields the well-known representation

$$
\begin{aligned}
& \boldsymbol{\Sigma}_{g}=\boldsymbol{\Lambda}_{g} \cdot \boldsymbol{\Phi}_{g} \cdot \boldsymbol{\Lambda}_{g}^{\top}+\boldsymbol{\Theta}_{g} \\
& \mathrm{E}\left(\boldsymbol{y}_{g}\right)=\boldsymbol{\alpha}_{g}+\boldsymbol{\Lambda}_{g} \cdot \boldsymbol{\tau}_{g}
\end{aligned}
$$

of the covariance matrix of the manifest variables and the respective manifest means in group $g$. In this representation, $\boldsymbol{\Sigma}_{g}$ is the $p \times p$ covariance matrix of the manifest variables, $\boldsymbol{\Phi}_{g}$ is the $p \times p$ covariance matrix of the latent variables, $\boldsymbol{\Theta}_{g}$ is a $p \times p$ diagonal matrix with the manifest residual variances, and $\boldsymbol{\tau}_{g}$ is the $n \times 1$ vector of latent means.

In this article with its focus on metric MI, we only consider the covariance matrix $\boldsymbol{\Sigma}_{g}$ as the meanstructure is not relevant for our considerations. That means, we consider $\boldsymbol{\alpha}_{g}$ and $\boldsymbol{\tau}_{g}$ as given under a ceteris paribus condition and rest our further considerations only on $\boldsymbol{\Lambda}_{g}, \boldsymbol{\Phi}_{g}$, and $\boldsymbol{\Theta}_{g}$. We collect the relevant matrices per group in the parameter vector $\theta_{g}=\left(\boldsymbol{\Lambda}_{g}, \boldsymbol{\Phi}_{g}, \boldsymbol{\Theta}_{g}\right)$ and denote the numerical values that the model parameters take in the population as data generating process $(\mathrm{dgp})$. We denote the estimated parameters with $\widehat{\theta}_{g}=\left(\widehat{\boldsymbol{\Lambda}}_{g}, \widehat{\boldsymbol{\Phi}}_{g}, \widehat{\boldsymbol{\Theta}}_{g}\right)$. We use the term covariance matrix when we refer to the covariance matrix resulting from the population parameters $\theta$ and we use the term model implied covariance matrix when we

refer to the covariance matrix resulting from the estimated parameters $\widehat{\theta}$. Moreover, as an important restriction, we only consider independent cluster models, i.e., we only consider models without cross-loadings. 
In their example, Raykov et al. (2020) used $p=12$ manifest variables and one latent variable $\eta_{G}, n=1$. The actual population model parameters are:

$$
\begin{aligned}
& \boldsymbol{\Lambda}_{1}=(1,1.25,1.25,1.25,1.5,1.5,1.5,1.5,1.75,1.75,1.75,2)^{\top}, \\
& \boldsymbol{\Lambda}_{2}=(1,1.25,1.25,1.25,1.5,1.5,1.5,1.5,1.75,1.75,1.75,2.5)^{\top}, \\
& \phi_{1,1,1}=\phi_{1,1,2}=1, \\
& \theta_{i, i, g}=2,1 \leq i \leq p, \\
& \boldsymbol{\alpha}_{1}=\boldsymbol{\alpha}_{2}=(1,1.25,1.25,1.25,1.5,1.5,1.5,1.5,1.75,1.75,1.75,2)^{\top}, \\
& \boldsymbol{\tau}_{1}=\boldsymbol{\tau}_{2}=\mathbf{0} .
\end{aligned}
$$

The last indicator is non-invariant with $\lambda_{12,1,1}=2$ in the reference group and $\lambda_{12,1,2}=2.5$ in the focal group. We consider the population parameters in (4) as the data generating process dgp. As in this example, the model includes the indicators' intercepts and the factor mean, there are 130 degrees of freedom for the scaled metric model. For the usual $\alpha=.05$ significance level, the critical value of the $\chi^{2}$-test statistic to reject the metric MI model is $\chi_{c r i t}^{2}=157.610$. As the model violates metric MI, the metric MI models' $\chi^{2}$-test statistics would be expected to be significant.

Raykov et al. (2020) conducted a Monte Carlo simulation with 10,000 repetitions and a sample size of $N=400$. They used FM scaling and the non-invariant indicator as referent indicator. The authors reported an average $\chi^{2}$-test statistic of the MI model of 150.394 $(S D=18.205)$. This average is below the critical value of 157.610 and therefore, in this example the model would on average fail to reject the violation of metric MI.

Now, we continue with the example of Raykov et al. (2020) and investigate how the other scaling methods perform in the same setting. Especially, we use all variants of the FM scaling, i.e., we use every indicator as the referent indicator and also use the EC and RG scaling methods. The results are shown in Table 1. Obviously, each scaling method results in the same average $\chi^{2}$-test statistic and therefore, fails to reject the faulty metric MI model. This equality of the average $\chi^{2}$-test statistic across all scaling methods yields the question if only the summary statistics are equal or if the $\chi^{2}$-test statistic is equal for each run of the Monte Carlo simulation under each scaling methods. Table 2 answers this question: The table shows the $\chi^{2}$-test statistic, its $d f$ and $p$-value as well as the log-likelihood under the $H_{0}$ for the first three Monte Carlo runs under each scaling methods. This table reveals that not only summary statistics are equal but that the $\chi^{2}$-statistic is equal for each run under each scaling method. 
Especially, this result corroborates the findings of Johnson et al. (2009) that regardless of the choice of the referent indicator in FM scaling, the same average $\chi^{2}$-test statistic results. Moreover, for the EC and RG scaling the same average $\chi^{2}$-test statistic is also observed. This result raises to two questions. Firstly, the question arises why this is the case and secondly, why the model fails to detect the violation of metric MI? In the following, we want to elaborate on the first question.

Concerning scaling methods, Klopp and Klößner (in press) provided a framework that allows us to study what happens in the case of this example. To make the following considerations easier to follow, we change from the simulation studies to a specific example, i.e., we use the example given by Raykov et al. (2020) on page 5. This example builds on a draw with a sample size of $N=400$ from the population with the dgp in (4). Again, we estimate the metric MI model with all scaling methods. The parameter estimates of the model are given in Table 3. From the results of the Monte Carlo simulations above, we expect that the estimated model has the same $\chi^{2}$-test statistic under all scaling methods. The results confirm this expectation, the model's test statistic is $\chi^{2}=112.279, d f=130, p=.867$, irrespective of the scaling method. Thus, in this sample, the model also fails to reject the violation of metric MI. Moreover, a further expectation resulting from the fact that the $\chi^{2}$-test statistics for each run under each scaling method is that the model-implied covariance matrices for each group $g$ coincide, i.e., $\Sigma\left(\widehat{\theta}_{g}^{(\mathrm{FM} 1)}\right)=\cdots=\Sigma\left(\widehat{\theta}_{g}^{(\mathrm{FM} 12)}\right)=\boldsymbol{\Sigma}\left(\widehat{\theta}_{g}^{(\mathrm{EC})}\right)=\boldsymbol{\Sigma}\left(\widehat{\theta}_{g}^{(\mathrm{RG})}\right)$. Again, this expectation is confirmed, the model implied covariance matrix is shown in Table $4 .^{3}$

In their second proposition, Klopp and Klößner (in press) provide equations which allow the conversion of estimated parameters obtained under any scaling method to the estimates obtained under any other scaling method without the need to re-estimate the model. The condition that must hold is that the model-implied covariance matrices under both scaling methods are equal. This condition holds in the current example and the conversion equations are applicable. This, in turn, means that we can convert the parameter estimates, however in each group separately. In the following, we exemplify the conversion of the estimates obtained under any scaling method $S$, which denotes any scaling method from the set $\mathcal{S}=\{\mathrm{FM} 1, \ldots, \mathrm{FM} 12, \mathrm{EC}, \mathrm{RG}\}$, to the estimates obtained under FM12 scaling. The conver-

\footnotetext{
${ }^{3}$ In Table 4, the model-implied covariance matrix for FM1 is shown, all other model-implied covariances matrices can be found in the supplementary material on OSF. As can be checked there, all the model-implied covariance matrices essentially coincide. The negligible differences sometimes observable stem from the fact that for determining the maximum likelihood estimates, an iterative search is employed which does not calculate the ML estimates exactly, but only up to a given precision.
} 
sion equations for the one-factorial MI model are

$$
\begin{aligned}
\widehat{\lambda}_{i, 1}^{(\mathrm{FM} 12)} & =\frac{\widehat{\lambda}_{i, 1}^{(S)}}{\widehat{\lambda}_{12,1}^{(S)}} \\
\widehat{\phi}_{1,1, g}^{(\mathrm{FM} 12)} & =\left(\widehat{\lambda}_{12,1}^{(S)}\right)^{2} \cdot \widehat{\phi}_{1,1, g}^{(S)}
\end{aligned}
$$

where $1 \leq i \leq p$. Especially, please note that these formulas also contain the case when $S$ represents any other variant of the FM scaling, e.g., FM1. Note that the conversions for the other variants of the FM scaling method are analogous.

We illustrate this conversion and convert the estimated parameters under FM1 scaling to those obtained under FM12 scaling. In (5) and (6), we have to replace $S$ on the right-hand side with FM1. The estimated loadings and the estimated variance in the first group under FM1 can be seen in Table 3, column FM1, accordingly for FM12 in the column labeled FM12. We start with the conversion of the estimated loadings. As the loadings in the metric MI model are equal in both groups, this demonstration must only be done once. According to the conversion equations above, converting from FM1 into FM12 requires dividing the estimated loadings obtained under FM1 by $\widehat{\lambda}_{12,1}^{(\mathrm{FM} 1)}$, i.e. $\widehat{\lambda}_{i, 1}^{(\mathrm{FM} 12)}=\frac{\widehat{\lambda}_{i, 1}^{(\mathrm{FM} 1)}}{\widehat{\lambda}_{12,1}^{(\mathrm{FM} 1)}}$. Obviously, this division shows that $\hat{\lambda}_{1,12}^{(\mathrm{FM} 12)}=\frac{2.521}{2.521}=1$ equals exactly the restriction of the estimated loading under FM12. For the first indicator, the conversion is $\widehat{\lambda}_{1,1}^{(\mathrm{FM} 12)}=\frac{1.000}{2.521}=0.397$ and for the second indicator, the conversion is $\widehat{\lambda}_{1,2}^{\text {(FM12) }}=\frac{1.380}{2.521}=0.547$. It is easy to verify that these converted loadings correspond to the estimates directly obtained under FM12. To convert the variances, we have to use the formula $\widehat{\phi}_{1,1, g}^{(\mathrm{FM} 12)}=\left(\widehat{\lambda}_{12,1}^{(\mathrm{FM} 1)}\right)^{2} \cdot \widehat{\phi}_{1,1, g}^{(\mathrm{FM} 12)}$. In contrast to the estimated loadings, the estimated variances differ between the groups; thus, the conversion has to be done in each group. In the referent group, we have $\widehat{\phi}_{1,1,1}^{(\mathrm{FM} 12)}=2.521^{2} \cdot 0.657=4.176$ and in the focal group, we have $\widehat{\phi}_{1,1,2}^{(\mathrm{FM} 12)}=2.521^{2} \cdot 0.742=4.716 \approx 4.717$. We leave further conversions between the estimated loadings of FM1 and FM12 to the reader. ${ }^{4}$

For the conversion from any scaling method $S$ to EC scaling, the conversion equations are

$$
\begin{aligned}
\widehat{\lambda}_{i, 1}^{(\mathrm{EC})} & =\frac{\widehat{\lambda}_{i, 1}^{(S)}}{\left.\mathrm{A}\left(\widehat{\lambda}_{\bullet, 1}\right)^{(S)}\right)} \\
\widehat{\phi}_{1,1, g}^{(E C)} & \left.=\left(\mathrm{A}\left(\widehat{\lambda}_{\bullet, 1}\right)^{(S)}\right)\right)^{2} \cdot \widehat{\phi}_{1,1, g}^{(S)}
\end{aligned}
$$

\footnotetext{
${ }^{4}$ We want to remark that with the conversion, there is a rounding problem. As Mplus provides the parameters loadings with three decimal places, rounding errors may occur in the third decimal place and sometimes in the second decimal place. However, this does not falsify the appropriateness of the conversion equations.
} 
where $\left.\mathrm{A}\left(\widehat{\lambda}_{\bullet, 1}\right)^{(S)}\right)$ is the mean of the estimated loadings, and for the conversion to RG scaling the conversion equations are

$$
\begin{aligned}
& \widehat{\lambda}_{i, 1}^{(\mathrm{RG})}=\sqrt{\widehat{\phi}_{1,1,1}^{S}} \cdot \widehat{\lambda}_{i, 1}^{(S)} \\
& \widehat{\phi}_{1,1, g}^{(\mathrm{RG})}=\frac{\sqrt{\widehat{\phi}_{1,1, g}^{S}}}{\sqrt{\widehat{\phi}_{1,1,1}^{S}}} .
\end{aligned}
$$

Obviously, equation (10) shows that the conversion gives the necessary restriction on the estimated variance in the referent group and leaves the estimated variance free in the focal group.

Carefully examining the conversion equations (5) to (10) reveals that they all have a common structure. To start with, we note that the scaling method that was actually used has no effect on the conversion, only the "target" scaling method is of relevance (Klopp \& Klößner, in press). For the further considerations, let $S_{1} \in \mathcal{S}$ be any scaling method and the one that was actually used in the estimation, let $S_{2} \in \mathcal{S} \backslash\left\{S_{1}\right\}$ be any other scaling method which is at the same time the "target" scaling method, and let $c \in \mathbb{R} \backslash\{0\}$ be a constant. Then the general conversion equations are

$$
\begin{aligned}
& \widehat{\lambda}_{i, i}^{\left(S_{2}\right)}=\frac{1}{c} \cdot \widehat{\lambda}_{i, i}^{\left(S_{1}\right)} \\
& \widehat{\phi}_{i, i, g}^{\left(S_{2}\right)}=c^{2} \cdot \widehat{\phi}_{i, i, g}^{\left(S_{1}\right)} .
\end{aligned}
$$

Obviously, choosing either $\left.c=\widehat{\lambda}_{i, R}^{\left(S_{1}\right)}, c=\mathrm{A}\left(\widehat{\lambda}_{\bullet, i,}\right)^{\left(S_{1}\right)}\right)$, or $c=\frac{1}{\sqrt{\widehat{\phi}_{1,1,1}^{S_{1}}}}$ gives the conversion equations and in general, we have

$$
\begin{aligned}
\Sigma\left(\widehat{\theta}_{g}^{\left(S_{2}\right)}\right) & =\widehat{\Lambda}^{\left(S_{2}\right)} \cdot \widehat{\Phi}_{g}^{\left(S_{2}\right)} \cdot \widehat{\Lambda}^{\left(S_{2}\right) \top}+\Theta_{g}= \\
& =\frac{1}{c} \cdot \widehat{\Lambda}^{\left(S_{1}\right)} \cdot\left(c^{2} \cdot \widehat{\Phi}_{g}^{\left(S_{1}\right)}\right) \cdot \frac{1}{c} \cdot \widehat{\Lambda}^{\left(S_{1}\right)}+\Theta_{g} \\
& =\widehat{\Lambda}^{\left(S_{1}\right)} \cdot \widehat{\Phi}_{g}^{\left(S_{1}\right)} \cdot \widehat{\Lambda}^{\left(S_{1}\right) \top}+\Theta_{g}=\boldsymbol{\Sigma}\left(\widehat{\theta}_{g}^{\left(S_{1}\right)}\right)
\end{aligned}
$$

The relation in Equation (13) is widely known in the SEM literature (e.g., Jöreskog (1978); Klopp and Klößner (in press); Mulaik (2010, p. 443); SanMartin, Gonzalez, and Tuckerlinckx (2014); Schweizer (2011); Schweizer, Troche, and DiStefano (2019); Schweizer and Troche (2019); von Oertzen (2010); Yoon and Millsap (2007)). In this context, Equation (13) indicates that for any parameter vector $\widehat{\theta}^{\left(S_{1}\right)}$ estimated under the $S_{1}$ scaling method, there is an equivalent vector of estimated parameters $\widehat{\theta}^{\left(S_{2}\right)}$ which yields the same model-implied covariance matrix. In other words, for any estimated parameter vector that fulfills the scaling 
restrictions of $S_{1}$, there is an equivalent estimated parameter vector that fulfills the scaling restrictions of $S_{2}$ such that the same model-implied covariance matrix results.

However, to finally explain why the same $\chi^{2}$-test statistic arises, an additional step is necessary. For the following considerations, we start with considering the estimated parameters under $S_{1}$, i.e., $\widehat{\theta}^{\left(S_{1}\right)}$. For the parameter vector $\widehat{\theta}^{\left(S_{1}\right)}$, we know that it minimizes the discrepancy function $F_{(\mathrm{ML})}\left(\widehat{\theta}^{\left(S_{1}\right)}\right)$ given the constraints of the scaling method $S_{1}$ and the constraint $\boldsymbol{c}=\widehat{\boldsymbol{\Lambda}}_{1}^{\left(S_{1}\right)}-\widehat{\boldsymbol{\Lambda}}_{2}^{\left(S_{1}\right)}=\mathbf{0}$. On the other hand, we know that there is also an estimated parameter vector which fulfills the constraints of the scaling method $S_{2}$ and the constraint $\boldsymbol{c}=\mathbf{0}$ and for which the discrepancy function takes the same value as for $\widehat{\theta}^{\left(S_{1}\right)}$. Therefore, the parameter vector $\widehat{\theta}^{\left(S_{2}\right)}$ minimizes the discrepancy function under all parameter vectors which fulfill the constraints of the scaling method $S_{2}$ and the constraint $\boldsymbol{c}=\mathbf{0}$. However, there may be other parameter vectors in the set fulfilling the constraints of the scaling method $S_{2}$ and the constraint that provides a "better" solution to the minimization of the discrepancy function. We therefore have

$$
F_{(\mathrm{ML})}\left(\widehat{\theta}^{\left(S_{1}\right)}\right) \geq F_{(\mathrm{ML})}\left(\widehat{\theta}^{\left(S_{2}\right)}\right) .
$$

By interchanging the roles of $S_{1}$ and $S_{2}$, we then find

$$
F_{(\mathrm{ML})}\left(\widehat{\theta}^{\left(S_{2}\right)}\right) \geq F_{(\mathrm{ML})}\left(\widehat{\theta}^{\left(S_{1}\right)}\right)
$$

Taking Equations (14) and (15) together, we have

$$
F_{(\mathrm{ML})}\left(\widehat{\theta}^{\left(S_{1}\right)}\right)=F_{(\mathrm{ML})}\left(\widehat{\theta}^{\left(S_{2}\right)}\right)
$$

showing that the same $\chi^{2}$-test statistic arises under the various scaling methods.

The derivation above explains why the $\chi^{2}$-test statistic or, equivalently, the minimum of the discrepancy function of the metric MI model take the same value for all scaling methods. This result also extends the finding of the simulation study by Johnson et al. (2009) that the choice of non-invariant referent indicators does not affect tests of metric MI. In summary, the derivation above shows that the choice of a particular referent indicator using FM scaling or the choice of the scaling method in general does not affect the metric MI model's $\chi^{2}$-test statistic. In consequence, the choice of the referent indicator in particular or the choice of scaling method in general does not affect the metric MI model's ability to detect deviations from metric MI. Before we continue to further scrutinize why, in the example above, the $\chi^{2}$-test statistic fails on average to indicate the lack of MI in the model, we want to mention an important restriction in the above considerations. The given derivation does not apply in the case of a partial metric MI model. In such a setting, it may happen that depending 
on the choice of the referent indicator or the scaling method, a different $\chi^{2}$-test statistic will result, i.e., a phenomenon called constraint interaction might show up (Klößner \& Klopp, 2019; Steiger, 2002). As the focus in this paper is on the full metric MI model, we do not elaborate further on this issue and now turn to the question which factors affect the $\chi^{2}$-test statistic.

\section{Factors affecting the $\chi^{2}$-test statistic and the signal-to- noise ratio}

As it is well known, sample size directly relates with the $\chi^{2}$-test statistics if the model is misspecified. Let $F_{(\mathrm{ML})}$ be the minimum of the discrepancy function and $N$ the sample size, then, according to Kline $(2016 \text {, p. } 207)^{5}$ the $\chi^{2}$-test statistic is given by

$$
\chi^{2}=2 \cdot N \cdot F_{(\mathrm{ML})} .
$$

Thus, with increasing sample size, the $\chi^{2}$-test statistics increases. However, if the model is correctly specified, then the $\chi^{2}$-test statistic would be around its expected value, which is equal to its degrees of freedom.

Besides this relation with sample size, in the context of measurement invariance analysis, Meade and Bauer (2007) summarized research (Hogarty, Hibes, Kromrey, \& Mumford, 2005; Gerbing \& Anderson, 1987; Marsh, Hau, Balla, \& Grayson, 1998; MacCallum, Widaman, Zhang, \& Hong, 1999) demonstrating that the precision of estimated factor loadings increases as sample size increases. Increasing precision of the estimated loadings in turn is also a reduction of noise, which is, this time, caused by the decrease of sample fluctuation and should therefore also yield the metric MI model to better detect indicators violating MI. However, a factor that counteracts is the size of the manifest residual variances $\Theta_{g}$. Heene et al. (2011) demonstrated that the manifest residual variances contribute to the size of the $\chi^{2}$ test statistic and can mask misfit of misspecified models. According to Browne, MacCallum, Kim, Andersen, and Glaser (2002), the $\chi^{2}$-test statistic is more sensitive to detect misfit when manifest residual variances are small than when they are large. Especially, these authors demonstrated that the manifest residual variances and the $\chi^{2}$-test statistic are oppositely related; when the manifest residual variances decreases, then the $\chi^{2}$-test statistic increases. Therefore, a factor contributing to the failure to detect the deviation from metric MI may

\footnotetext{
${ }^{5}$ As we used Mplus, the relation between the $\chi^{2}$-test statistic and the discrepancy function calculated in this software is according to Equation (17), see Muthén (2004, p. 21).
} 
therefore have been large manifest residual variances. However, the question what is a large or a small manifest residual variance remains.

In psychology and other social sciences, an unobserved latent variable reflects an underlying cause that yields the covariation between the observed manifest variables (e.g., Borsboom, Mellenbergh, \& Heerden, 2003). This covariation is represented by the covariance matrix $\boldsymbol{\Sigma}_{g}$. According to Equation (2), the variance of the indicator $y_{i}, 1 \leq i \leq p$, loading on the latent variable $\eta_{j}, 1 \leq j \leq n$, in the group $g$ is given by

$$
\operatorname{var}\left(y_{i, j, g}\right)=\underbrace{\left(\lambda_{i, j, g}\right)^{2} \cdot \phi_{j, j, g}}_{=: h_{i, j, g}^{2}}+\theta_{i, i, g} .
$$

The first term on the right-hand-side represents the indicator communality $h_{i, j, g}^{2}$. Indicator communality $h_{i, j, g}^{2}$ refers to the portion of an indicator's variance that is due to the latent variable (Mulaik, 2010, p. 134). The second term denotes the portion of variance that is due to the manifest measurement error. In general, this error variance may contain systematic error as well as random error. In the following, we assume that we only have random error.

From another point of view, latent variables form a signal that causes the observed manifest data, or respectively, its covariation. In addition to the signal, there is some independent background noise that additively contributes to the manifest variables (Owen \& Wang, 2016). The signal is generated by the latent variables whereas the background noise is generated by random errors. Thus, the variation of the signal is indicated by the indicator communality. By defining the ratio of indicator communality over manifest residual variance, we receive a measure that is similar to the concept of signal-to-noise-ratio (SNR). The SNR indicates how noisy a measurement is and is given by the ratio of the power of a signal over the power of background noise. The power of signal and the power of the background noise may be indicated by the variances of these measurements. In the context of latent variable models, the variance that is due to the latent variable can be considered as power of a signal, i.e., meaningful information, and the variance that is due to measurement error can be considered as noise, i.e., unwanted background noise. Thus, we can define the SNR for indicator $y_{i}$, $1 \leq i \leq p$, and the latent variable $\eta_{j}, 1 \leq j \leq n$, in group $g$ as

$$
\mathrm{SNR}_{i, j, g}:=\frac{h_{i, j, g}^{2}}{\theta_{i, i, g}}=\frac{\left(\lambda_{i, j, g}\right)^{2} \cdot \psi_{j, j, g}}{\theta_{i, i, g}} .
$$

Obviously, according to the preceding formula, when the manifest residual variance is greater than the indicator communality, then $\mathrm{SNR}_{i, j, g}<1$, and the measurement is considered a rather noisy one. Vice versa, when indicator communality is greater than manifest residual 
variance, $\mathrm{SNR}_{i, j, g}>1$, then the power of the signal is greater than the power of the background noise. A $\mathrm{SNR}_{i, j, g}=1$ indicates that the power of the signal equals the power of the noise. Hereafter, we consider a threshold $\mathrm{SNR}_{i, j, g} \geq 1.5$ as reflecting an amount of variance due to the latent variable that is considerably greater than 1 . This threshold represents the belief that the signal should be at least $50 \%$ larger than the noise.

Drawing on the reasoning above, we extended the example of Raykov et al. (2020) and varied the sample size and the manifest residual variance. Firstly, according to (17), we expect that with increasing sample size, the average of the $\chi^{2}$-test statistic increases. Secondly, according to Browne et al. (2002), we also expect that with decreasing manifest residual variances, the average of the $\chi^{2}$-test statistic increases. We varied the sample size with $N \in\{200,400,600,800,1000\}$ and the manifest residual variances with $\theta_{i, i, g} \in\{0.5,1,2,4\}$ for all indicators, yielding a total of 20 conditions. Table 5 depicts the resulting population SNRs in all conditions. To scale the latent variable, we used FM1 (because of the equivalence of all scaling methods we do not consider the other variations of FM and the two other scaling methods). In order to explore the effects of manifest residual variance and sample size, we consider the average of the metric MI models' $\chi^{2}$-test statistic over all replications and conducted a Monte Carlo study.

The results are presented in Figure 1 and Table 6. They show that the average of the $\chi^{2}-$ test statistics depends on the manifest residual variances and the sample size. For $\theta_{i, i, g}=4$, which is the condition with the largest manifest residual variances, Figure 1 shows that for all sample sizes the average of the $\chi^{2}$-test statistics is below the critical $\chi_{c r i t}^{2}$-value. For $\theta_{i, i, g}=2$, starting with a sample size of $N=600$, the average of the $\chi^{2}$-test statistic is above the critical $\chi_{c r i t}^{2}$-value. For $\theta_{i, i, g}=1$, Figure 1 reveals that only for the smallest sample size of $N=200$ the average of the $\chi^{2}$-test statistic is below the critical $\chi_{c r i t}^{2}$-value, and for $\theta_{i, i, g}=0.5$, which is the condition with the smallest manifest residual variances, the average of the $\chi^{2}$-test statistic is above the critical $\chi_{\text {crit }}^{2}$-value for all samples sizes.

We now use the concept of SNR and equation (19) to study the simulation results from the previous section, see Table 5 . This table shows the SNRs for the various settings of $\theta_{i, i, g}$. For the conditions with $\theta_{i, i, g}=4$, only the last, non-invariant, indicator in the focal group has a SNR that is just above 1.5. For $\theta_{i, i, g}=2$, a third of indicators have SNRs that are considerably larger than 1 in both groups. In the conditions with $\theta_{i, i, g}=1$, all but the first indicator have SNRs considerably greater than 1 in both groups. Finally, in the condition with $\theta_{i, i, g}=0.5$, all indicators in both groups have SNRs considerably larger than 1. This consideration mirrors the results from the Monte Carlo study. As the SNR of the non-invariant indicator and the SNRs of the other indicators increase, the average of the metric MI models' $\chi^{2}$-test statistic increases as well. 
Taken together, the results demonstrate the well-known relation of sample size and $\chi^{2}$-test statistic: the greater the sample size the greater the average $\chi^{2}$-test statistic if the model is misspecified. In addition, the results demonstrate that there is an inverse relation between manifest residual variances and $\chi^{2}$-test statistic: the greater the manifest residual variances the smaller the average $\chi^{2}$-test statistic. Stated otherwise, the metric MI models' ability to detect the violation of equal loadings in both groups depends not only on the sample size, which is quite obvious and relates to the question of power, but also on the size of the manifest residual variances, a quantity which is commonly not considered in the way outlined in this paper when metric MI is analyzed. ${ }^{6}$

\section{Discussion}

In this contribution we used an example introduced by Raykov et al. (2020) to explain why, when testing for metric MI, all known scaling methods yield the same $\chi^{2}$-test statistic and explained the reason for this finding. Moreover, we demonstrated that the size of the manifest residual variances is the cause that the $\chi^{2}$-test statistic does not detect the deviation from metric MI in the example. As a measure of the size of the manifest residual variances we introduced the SNR. As the SNR is defined as the ratio of indicator communality over the manifest residual variance of the indicators, it focuses on quantities that are only seldom reported in MI analyses.

Although the example in this contribution demonstrated the effects of indicator communalities, the investigated model does not represent the type of models typically investigated in practical applications. Usually, there are more than one factor and more than two groups. Further studies should therefore target on more complex and more realistic settings.

Notwithstanding these limitations, it is obvious that the reasoning put forward in this article easily generalizes to more complex settings. Regarding more complex factor structures, the usual setting in a multiple-group confirmatory factor analysis setting is an independent cluster model in which each indicator only loads on one latent variable and therefore constitutes a simple structure. In such structures the SNR is always calculated according to Equation (19) because of the absence of cross-loadings. In the same way as for the number of groups, the confirmatory factor analysis models can be broken down in one-factor models. In summary, although we only considered a simple dgp, the results are valid to highlight the importance of indicator communalities and manifest residual variances in the analysis of metric MI.

\footnotetext{
${ }^{6}$ Usually, manifest residual variances only matter in measurement in variance analysis when they are tested for equality.
} 
Two further limitations apply to the Monte Carlo studies in this contribution. Firstly, in all MC studies we only considered the ML estimator. It is obvious that different estimators yield different effects of indicator communality and manifest residual variances on the average of the $\chi^{2}$-test statistic for the metric MI model. Further studies should therefore consider different estimators. Secondly, the main focus in this contribution was on the $\chi^{2}$-test statistic for the metric MI model. As mentioned in the introduction, the analysis of MI consists in a series of nested model comparisons. Thus, the question for the average of the $\chi^{2}$-differencetest is obvious. Further studies should also include the typically considered set of fit indices, i.e., the CFI, SRMR and RMSEA.

As a last limitation, we want to mention that we only studied the average behavior of the $\chi^{2}$-test statistic. This was implemented by comparing the average of the $\chi^{2}$-test statistic to its critical value. Further studies should consider the power of the metric MI models' $\chi^{2}$-test directly. Moreover, further studies should also take the $\chi^{2}$-difference test between the configural and metric MI model and its power into account.

Regarding practical applications, an important feature in this paper was the use of Monte Carlo studies. In this setting, the dgp in the population is known and we calculated the SNRs drawing on the population quantities of the parameters. This is not possible in applied settings. In applied settings, the estimated parameters have to be used. To get unique parameters, the model has to be scaled. As the SNRs are calculated in the configural model, where there are no across-group equality constraints, each group is scaled separately (usually by means of the same scaling method). In such a setting, the estimated SNR is invariant with respect to the choice of a particular scaling method. The product of estimated loading times estimated latent variance is invariant, which is easily shown with the conversion equation in their general form, and the manifest residual variances are always estimated as their population counterpart (as long as the model is correctly specified, Klößner \& Klopp, 2019; Klopp \& Klößner, in press).

Moreover, this contribution also questions the usual reporting practice. In almost all cases of MI analyses, the authors report the results for the nested model comparison and common reliability indices as a measure for the scale's quality. Drawing on the simulation studies in this contribution, it seems necessary to report the SNR, or the quantities involved in calculating the SNR, i.e., the loadings, latent variance and manifest residual variances in the configural model so that a judgment regarding the power can be made. A more elaborate way would be not only to report the SNR, but to use the results from a given metric MI model as dgp and evaluate the power of the model using a simulation. 


\section{References}

Bollen, K. A. (1989). Structural equations with latent variables. New York: Wiley.

Borsboom, D., Mellenbergh, G., \& Heerden, J. (2003). The theoretical status of latent variables. Psychological review, 110, 203-19.

Brown, T. A. (2015). Confirmatory factor analysis for applied research (2. ed.). New York: The Guilford Press.

Browne, M. W., MacCallum, R. C., Kim, C.-T., Andersen, B. L., \& Glaser, R. (2002). When fit indices and residuals are incompatible. Psychological Methods, 7(4), 403 - 421.

Chen, F. F. (2007). Sensitivity of goodness of fit indexes to lack of measurement invariance. Structural Equation Modeling: A Multidisciplinary Journal, 14, 464-504.

Cheung, G. W., \& Rensvold, R. B. (1999). Testing factorial invariance across groups: A reconceptualization and proposed new method. Journal of Management, 25(1), 1-27.

Cheung, G. W., \& Rensvold, R. B. (2002). Evaluating goodness-of-fit indexes for testing measurement invariance. Structural Equation Modeling: A multidisciplinary Journal, $9(2), 233-255$.

Davidov, E., Muthen, B., \& Schmidt, P. (2018). Measurement invariance in cross-national studies: Challenging traditional approaches and evaluating new ones. Sociological Methods $\&$ Research, 47, 631-636.

Gerbing, D. W., \& Anderson, J. C. (1987). Improper solutions in the analysis of covariance structures: Their interpretability and a comparison of alternate respecifications. Psychometrika, 52, 99-111.

Greiff, S., \& Scherer, R. (2018). Still comparing apples with oranges. European Journal of Psychological Assessment, 34, 141-144.

Heene, M., Hilbert, S., Draxler, C., Ziegler, M., \& Bühner, M. (2011). Masking misfit in confirmatory factor analysis by increasing unique variances: A cautionary note on the usefulness of cutoff values of fit indices. Psychological Methods, 16, 319-336.

Hogarty, K. Y., Hibes, C. V., Kromrey, J. D., \& Mumford, K. R. (2005). The quality of factor solutions in exploratory factor analysis: The influence of sample size, communality, and overdetermination. Educational and Psychological Measurement, 65, 206-226.

Holland, P. W., \& Thayer, D. T. (1988). Test validity. In H. Wainer \& H. Braum (Eds.), Differential item performance and the Mantel-Haenszel procedure (p. 129-145). New York: Hillsdale.

Jiang, G., Mai, Y., \& Yuan, K.-H. (2017). Advances in measurement invariance and mean comparison of latent variables: Equivalence testing and a projection-based approach. Frontiers in Psychology, 8, 1823. 
Johnson, E. C., Meade, A. W., \& DuVernet, A. M. (2009). The role of referent indicators in tests of measurement invariance. Structural Equation Modeling: A Multidisciplinary Journal, 16, 642-657.

Jöreskog, K. (1978). Structural analysis of covariance and correlation matrices. Psychometrika, 43, 443-477.

Kline, R. B. (2016). Principles and practice of structural equation modeling (Fourth ed.). New York: The Guilford Press.

Klopp, E., \& Klößner, S. (in press). The impact of the choice of scaling method on the properties and interpretation of parameter estimates in structural equation models with latent variables. Structural Equation Modeling: A Multidisciplinary Journal. Retrieved from https://osf.io/c9ke8/

Klößner, S., \& Klopp, E. (2019). Explaining constraint interaction: How to interpret estimated model parameters under alternative scaling methods. Structural Equation Modeling: A Multidisciplinary Journal, 26, 143-155.

Lee, J., Little, T. D., \& Preacher, K. J. (2011). Methodological issues in using structural equation models for testing differential item functioning. In E. Davidov, P. Schmidt, \& J. Billet (Eds.), Cross-cultural Analysis - Methods and Applications (p. 55-84). New York: Psychology Press.

Little, T. D., Slegers, D. W., \& Card, N. A. (2006). A non-arbitrary method of identifying and scaling latent variables in sem and macs models. Structural Equation Modeling: A Multidisciplinary Journal, 13(1), 59-72. doi: 10.1207/s15328007sem1301\_3

MacCallum, R. C., Widaman, K. F., Zhang, S., \& Hong, S. (1999). Sample size in factor analysis. Psychological Methods, 4, 84-99.

Marsh, H. W., Hau, K. T., Balla, J. R., \& Grayson, D. (1998). Is more ever too much? the number of indicators per factor in confirmatory factor analysis. Multivariate Behavioral Research, 33, 181-220.

Meade, A., \& Bauer, D. (2007). Power and precision in confirmatory factor analytic tests of measurement invariance. Structural Equation Modeling: A Multidisciplinary Journal, $14(4), 611-635$.

Meade, A., Johnson, E., \& Braddy, P. (2008). Power and sensitivity of alternative fit indices in tests of measurement invariance. Journal of Applied Psychology, 93(3), 568-592.

Millsap, R. (2011). Statistical approaches to measurement invariance. New York: Routledge.

Mulaik, S. A. (2010). Foundations of factor analysis (2. ed.). Boca Raton: CRC Press.

Muthén, B. (2004). Mplus. technical appendices. Los Angeles: Muthén \& Muthén.

Muthén, B., \& Muthén, L. (1998-2019). Mplus user's guide. 8th edition. Los Angeles: Muthén \& Muthén. 
Owen, A., \& Wang, J. (2016). Bi-cross-validation for factor analysis. Statistical Science, 31, 119-139.

Raykov, T., Marcoulides, G. A., Harrison, M., \& Zhang, M. (2020). On the dependability of a popular procedure for studying measurement invariance: A cause for concern? Structural Equation Modeling: A Multidisciplinary Journal, 27(4), 649-656. doi: 10 $.1080 / 10705511.2019 .1610409$

SanMartin, E., Gonzalez, J., \& Tuckerlinckx, F. (2014). On the unidentifiablility of the fixed-effects 3PL model. Psychometrika. doi: 10.1007/S11336-014-9404-2

Schweizer, K. (2011). Scaling variances of latent variables by standardizing loadings: applications to working memory and the position effect. Multivariate Behavioral Research, 46, 938-955.

Schweizer, K., \& Troche, S. (2019). The EV scaling method for variances of latent variables. Methodology, 15, 175-184.

Schweizer, K., Troche, S. J., \& DiStefano, C. (2019). Scaling the variance of a latent variable while assuring constancy of the model. Frontiers in Psychology, 10, 887. doi: 10.3389/ fpsyg.2019.00887

Steiger, J. H. (2002). When constraints interact: a caution about reference variables, identification constraints, and scale dependencies in structural equation modeling. Psychological Methods, 7(2), 210-227.

van de Schoot, R., Lugtig, P., \& Hox, J. (2012). A checklist for testing measurement invariance. European Journal of Developmental Psychology, 9(4), 486-492. doi: 10.1080/17405629.2012.686740

van de Schoot, R., Schmidt, P., \& de Beuckelaer, A. (2015). Measurement invariance. Lousanne: Frontiers Media.

von Oertzen, T. (2010). Power equivalence in structural equation modelling. British Journal of Mathematical and Statistical Psychology, 63(2), 257-272.

Yoon, M., \& Millsap, R. (2007). Detecting violations of factorial invariance using databased specification searches: A Monte Carlo study. Structural Equation Modeling: A Multidisciplinary Journal, 14(3), 435-463. 


\section{Tables}

Table 1: Means and standard deviations of the Monte Carlo simulation of the metric MI model with all scaling methods.

\begin{tabular}{lccc}
\hline Scaling method & $\chi^{2} M$ & $d f$ & $\chi^{2} S D$ \\
\hline FM1 & 150.394 & 130 & 18.205 \\
FM2 & 150.394 & 130 & 18.205 \\
FM3 & 150.394 & 130 & 18.205 \\
FM4 & 150.394 & 130 & 18.205 \\
FM5 & 150.394 & 130 & 18.205 \\
FM6 & 150.394 & 130 & 18.205 \\
FM7 & 150.394 & 130 & 18.205 \\
FM8 & 150.394 & 130 & 18.205 \\
FM9 & 150.394 & 130 & 18.205 \\
FM10 & 150.394 & 130 & 18.205 \\
FM11 & 150.394 & 130 & 18.205 \\
FM12 & 150.394 & 130 & 18.205 \\
EC & 150.394 & 130 & 18.205 \\
RG & 150.394 & 130 & 18.205 \\
\hline
\end{tabular}


Table 2: Results for the first three Monte Carlo runs of the metric MI model for all scaling methods.

\begin{tabular}{lccccc}
\hline Scaling & Run & $\chi^{2}$ & $d f$ & $p$ & $H_{0} \log$ \\
\hline FM1 & 1 & 139.848 & 130 & 0.262 & -18137.550 \\
FM1 & 2 & 142.591 & 130 & 0.212 & -18028.464 \\
FM1 & 3 & 112.128 & 130 & 0.869 & -18034.866 \\
FM2 & 1 & 139.848 & 130 & 0.262 & -18137.550 \\
FM2 & 2 & 142.591 & 130 & 0.212 & -18028.464 \\
FM2 & 3 & 112.128 & 130 & 0.869 & -18034.866 \\
FM3 & 1 & 139.848 & 130 & 0.262 & -18137.550 \\
FM3 & 2 & 142.591 & 130 & 0.212 & -18028.464 \\
FM3 & 3 & 112.128 & 130 & 0.869 & -18034.866 \\
FM4 & 1 & 139.848 & 130 & 0.262 & -18137.550 \\
FM4 & 2 & 142.591 & 130 & 0.212 & -18028.464 \\
FM4 & 3 & 112.128 & 130 & 0.869 & -18034.866 \\
FM5 & 1 & 139.848 & 130 & 0.262 & -18137.550 \\
FM5 & 2 & 142.591 & 130 & 0.212 & -18028.464 \\
FM5 & 3 & 112.128 & 130 & 0.869 & -18034.866 \\
FM6 & 1 & 139.848 & 130 & 0.262 & -18137.550 \\
FM6 & 2 & 142.591 & 130 & 0.212 & -18028.464 \\
FM6 & 3 & 112.128 & 130 & 0.869 & -18034.866 \\
FM7 & 1 & 139.848 & 130 & 0.262 & -18137.550 \\
FM7 & 2 & 142.591 & 130 & 0.212 & -18028.464 \\
FM7 & 3 & 112.128 & 130 & 0.869 & -18034.866 \\
FM8 & 1 & 139.848 & 130 & 0.262 & -18137.550 \\
FM8 & 2 & 142.591 & 130 & 0.212 & -18028.464 \\
FM8 & 3 & 112.128 & 130 & 0.869 & -18034.866 \\
FM9 & 1 & 139.848 & 130 & 0.262 & -18137.550 \\
FM9 & 2 & 142.591 & 130 & 0.212 & -18028.464 \\
FM9 & 3 & 112.128 & 130 & 0.869 & -18034.866 \\
FM10 & 1 & 139.848 & 130 & 0.262 & -18137.550 \\
FM10 & 2 & 142.591 & 130 & 0.212 & -18028.464 \\
FM10 & 3 & 112.128 & 130 & 0.869 & -18034.866 \\
FM11 & 1 & 139.848 & 130 & 0.262 & -18137.550 \\
FM11 & 2 & 142.591 & 130 & 0.212 & -18028.464 \\
FM11 & 3 & 112.128 & 130 & 0.869 & -18034.866 \\
FM12 & 1 & 139.848 & 130 & 0.262 & -18137.550 \\
FM12 & 2 & 142.591 & 130 & 0.212 & -18028.464 \\
FM12 & 3 & 112.128 & 130 & 0.869 & -18034.866 \\
EC & 1 & 139.848 & 130 & 0.262 & -18137.550 \\
EC & 2 & 142.591 & 130 & 0.212 & -18028.464 \\
EC & 3 & 112.128 & 130 & 0.869 & -18034.866 \\
RG & 1 & 139.848 & 130 & 0.262 & -18137.550 \\
RG & 2 & 142.591 & 130 & 0.212 & -18028.464 \\
\hline & 3 & 112.128 & 130 & 0.869 & -18034.866 \\
\hline & & & & &
\end{tabular}


Table 3: Parameter estimates of the metric MI in the referent and focal group for all scaling methods and $g \in\{1,2\}$.

\begin{tabular}{|c|c|c|c|c|c|c|c|c|c|c|c|c|c|c|c|c|}
\hline & FM1 & FM2 & FM3 & FM4 & FM5 & FM6 & FM7 & FM8 & FM9 & FM10 & FM11 & FM12 & EC & RG & $\widehat{\theta}_{i, 1,1}$ & $\widehat{\theta}_{i, 1,2}$ \\
\hline$\widehat{\lambda}_{1,1}$ & .000 & .725 & 0.721 & 0.703 & 0.586 & 0.554 & 0.601 & 0.581 & 0.525 & 0.504 & 0.494 & 0.397 & 0.585 & 0.810 & $\mid 2.063$ & 2.159 \\
\hline$\widehat{\lambda}_{1,2}$ & 1.380 & 1.000 & 0.995 & 0.970 & 0.808 & 0.765 & 0.829 & 0.801 & 0.725 & 0.696 & 0.681 & 0.547 & 0.807 & 1.118 & 1.987 & 1.787 \\
\hline$\widehat{\lambda}_{1,3}$ & 1.386 & 1.005 & 1.000 & 0.975 & 0.812 & 0.768 & 0.833 & 0.805 & 0.728 & 0.699 & 0.685 & 0.550 & 0.811 & 1.124 & 1.951 & 2.244 \\
\hline$\widehat{\lambda}_{1,4}$ & 1.422 & 1.031 & 1.026 & 1.000 & 0.833 & 0.788 & 0.855 & 0.826 & 0.747 & 0.717 & 0.702 & 0.564 & 0.832 & 1.153 & 2.029 & 2.005 \\
\hline$\widehat{\lambda}_{1,5}$ & 1.707 & 1.237 & 1.232 & 1.200 & 1.000 & 0.946 & 1.026 & 0.992 & 0.896 & 0.861 & 0.843 & 0.677 & 0.999 & 1.384 & 2.006 & 2.255 \\
\hline$\widehat{\lambda}_{1,6}$ & 1.804 & 1.308 & 1.302 & 1.269 & 1.057 & 1.000 & 1.084 & 1.048 & 0.948 & 0.910 & 0.891 & 0.716 & 1.055 & 1.462 & 2.002 & 2.099 \\
\hline$\widehat{\lambda}_{1,7}$ & 1.664 & 1.206 & 1.200 & 1.170 & 0.975 & 0.922 & 1.000 & 0.967 & 0.874 & 0.839 & 22 & 0.660 & 0.973 & 1.349 & 1.880 & 2.108 \\
\hline$\widehat{\lambda}_{1,8}$ & 1.722 & 1.248 & 1.242 & 1.210 & 1.008 & 0.954 & 1.035 & 1.000 & 0.904 & 0.868 & 0.850 & 0.683 & 1.007 & 1.395 & 1.981 & 1.952 \\
\hline$\widehat{\lambda}_{1,9}$ & 1.904 & 1.380 & 1.374 & 1.339 & 1.115 & 1.055 & 1.144 & 1.106 & 1.000 & 0.960 & 0.941 & 0.755 & 1.114 & 1.544 & 2.218 & 2.304 \\
\hline$\widehat{\lambda}_{1,10}$ & 1.983 & 1.437 & 1.430 & 1.394 & 1.161 & 1.099 & 1.192 & 1.152 & 1.041 & 1.000 & 0.979 & 0.786 & 1.160 & 1.607 & 1.934 & 1.923 \\
\hline$\widehat{\lambda}_{1,11}$ & 2.025 & 1.467 & 1.460 & 1.424 & 1.186 & 1.122 & 1.217 & 1.176 & 1.063 & 1.021 & 1.000 & 0.803 & 1.184 & 1.641 & 2.048 & 1.952 \\
\hline$\widehat{\lambda}_{1,12}$ & 2.521 & 1.827 & 1.819 & 1.773 & 1.477 & 1.397 & 1.515 & 1.465 & 1.324 & 1.272 & 1.245 & 1.000 & 1.474 & 2.043 & 1.738 & 2.598 \\
\hline & 0.657 & 1.250 & 1.262 & 1.329 & 1.915 & 2.139 & 1.819 & 1.947 & 2.382 & 2.582 & 2.692 & 4.176 & 1.921 & 1.000 & & \\
\hline$\widehat{\phi}_{1,1,2}$ & 0.742 & 1.413 & 1.426 & 1.501 & 2.163 & 2.416 & 2.055 & 2.200 & 2.691 & 2.918 & 3.042 & 4.717 & 2.170 & 1.130 & & \\
\hline
\end{tabular}

Note. Please note that the last two columns of the table represent the estimated manifest residual variances of the respective indicator. These are equal under all scaling methods. 
Table 4: Model implied covariance matrices in both groups (see also Footnote 3).

\begin{tabular}{|c|c|c|c|c|c|c|c|c|c|c|c|c|}
\hline \multicolumn{13}{|c|}{ Reference group } \\
\hline & $y_{1}$ & $y_{2}$ & $y_{3}$ & $y_{4}$ & $y_{5}$ & $y_{6}$ & $y_{7}$ & $y_{8}$ & $y_{9}$ & $y_{10}$ & $y_{11}$ & $y_{12}$ \\
\hline$y_{1}$ & 2.720 & & & & & & & & & & & \\
\hline$y_{2}$ & 0.906 & 3.237 & & & & & & & & & & \\
\hline$y_{3}$ & 0.911 & 1.256 & 3.214 & & & & & & & & & \\
\hline$y_{4}$ & 0.934 & 1.289 & 1.295 & 3.357 & & & & & & & & \\
\hline$y_{5}$ & 1.122 & 1.547 & 1.555 & 1.595 & 3.921 & & & & & & & \\
\hline$y_{6}$ & 1.185 & 1.635 & 1.643 & 1.686 & 2.024 & 4.141 & & & & & & \\
\hline$y_{7}$ & 1.093 & 1.508 & 1.515 & 1.554 & 1.866 & 1.972 & 3.699 & & & & & \\
\hline$y_{8}$ & 1.131 & 1.560 & 1.568 & 1.608 & 1.931 & 2.040 & 1.882 & 3.928 & & & & \\
\hline$y_{9}$ & 1.251 & 1.726 & 1.734 & 1.779 & 2.136 & 2.257 & 2.082 & 2.154 & 4.600 & & & \\
\hline$y_{10}$ & 1.302 & 1.797 & 1.806 & 1.852 & 2.224 & 2.350 & 2.167 & 2.242 & 2.480 & 4.516 & & \\
\hline$y_{11}$ & 1.330 & 1.835 & 1.844 & 1.891 & 2.271 & 2.400 & 2.213 & 2.289 & 2.533 & 2.637 & 4.740 & \\
\hline$y_{12}$ & 1.656 & 2.285 & 2.296 & 2.355 & 2.828 & 2.988 & 2.756 & 2.851 & 3.154 & 3.284 & 3.353 & 5.914 \\
\hline \multicolumn{13}{|c|}{ Focal group } \\
\hline & $y_{1}$ & $y_{2}$ & $y_{3}$ & $y_{4}$ & $y_{5}$ & $y_{6}$ & $y_{7}$ & $y_{8}$ & $y_{9}$ & $y_{10}$ & $y_{11}$ & $y_{12}$ \\
\hline$y_{1}$ & 2.901 & & & & & & & & & & & \\
\hline$y_{2}$ & 1.024 & 3.200 & & & & & & & & & & \\
\hline$y_{3}$ & 1.029 & 1.420 & 3.670 & & & & & & & & & \\
\hline$y_{4}$ & 1.055 & 1.456 & 1.463 & 3.506 & & & & & & & & \\
\hline$y_{5}$ & 1.267 & 1.748 & 1.757 & 1.802 & 4.418 & & & & & & & \\
\hline$y_{6}$ & 1.339 & 1.848 & 1.856 & 1.904 & 2.286 & 4.515 & & & & & & \\
\hline$y_{7}$ & 1.235 & 1.704 & 1.712 & 1.756 & 2.108 & 2.228 & 4.162 & & & & & \\
\hline$y_{8}$ & 1.278 & 1.763 & 1.771 & 1.817 & 2.181 & 2.305 & 2.126 & 4.152 & & & & \\
\hline$y_{9}$ & 1.413 & 1.950 & 1.959 & 2.010 & 2.413 & 2.550 & 2.352 & 2.433 & 4.996 & & & \\
\hline$y_{10}$ & 1.471 & 2.030 & 2.040 & 2.093 & 2.512 & 2.655 & 2.449 & 2.533 & 2.802 & 4.841 & & \\
\hline$y_{11}$ & 1.502 & 2.073 & 2.083 & 2.137 & 2.565 & 2.711 & 2.500 & 2.587 & 2.861 & 2.979 & 4.994 & \\
\hline$y_{12}$ & 1.871 & 2.582 & 2.594 & 2.661 & 3.195 & 3.376 & 3.114 & 3.221 & 3.563 & 3.710 & 3.788 & 7.316 \\
\hline
\end{tabular}


Table 5: Signal-to-noise ratios for various $\theta_{i, i, g}$.

\begin{tabular}{|c|c|c|c|c|}
\hline & 0.500 & 1.000 & 2.000 & 4.000 \\
\hline $\mathrm{SNR}_{y 1,1}$ & 2.000 & 1.000 & 0.500 & 0.250 \\
\hline $\mathrm{SNR}_{y 2,1}$ & 3.125 & 1.562 & 0.781 & 0.391 \\
\hline $\mathrm{SNR}_{y 3,1}$ & 3.125 & 1.562 & 0.781 & 0.391 \\
\hline $\mathrm{SNR}_{y 4,1}$ & 3.125 & 1.562 & 0.781 & 0.391 \\
\hline $\mathrm{SNR}_{y 5,1}$ & 4.500 & 2.250 & 1.125 & 0.562 \\
\hline $\mathrm{SNR}_{y 6,1}$ & 4.500 & 2.250 & 1.125 & 0.562 \\
\hline $\mathrm{SNR}_{y 7,1}$ & 4.500 & 2.250 & 1.125 & 0.562 \\
\hline $\mathrm{SNR}_{y 8,1}$ & 4.500 & 2.250 & 1.125 & 0.562 \\
\hline $\operatorname{SNR}_{y 9,1}$ & 8.000 & 4.000 & 2.000 & 1.000 \\
\hline $\mathrm{SNR}_{y 10,1}$ & 8.000 & 4.000 & 2.000 & 1.000 \\
\hline $\mathrm{SNR}_{y 11,1}$ & 8.000 & 4.000 & 2.000 & 1.000 \\
\hline $\mathrm{SNR}_{y 12,1}$ & 8.000 & 4.000 & 2.000 & 1.000 \\
\hline $\mathrm{SNR}_{y 1,2}$ & 2.000 & 1.000 & 0.500 & 0.250 \\
\hline $\mathrm{SNR}_{y 2,2}$ & 3.125 & 1.562 & 0.781 & 0.391 \\
\hline $\mathrm{SNR}_{y 3,2}$ & 3.125 & 1.562 & 0.781 & 0.391 \\
\hline $\mathrm{SNR}_{y 4,2}$ & 3.125 & 1.562 & 0.781 & 0.391 \\
\hline $\mathrm{SNR}_{y 5,2}$ & 4.500 & 2.250 & 1.125 & 0.562 \\
\hline $\mathrm{SNR}_{y 6,2}$ & 4.500 & 2.250 & 1.125 & 0.562 \\
\hline $\mathrm{SNR}_{y 7,2}$ & 4.500 & 2.250 & 1.125 & 0.562 \\
\hline $\mathrm{SNR}_{y 8,2}$ & 4.500 & 2.250 & 1.125 & 0.562 \\
\hline $\mathrm{SNR}_{y 9,2}$ & 8.000 & 4.000 & 2.000 & 1.000 \\
\hline $\mathrm{SNR}_{y 10,2}$ & 8.000 & 4.000 & 2.000 & 1.000 \\
\hline $\mathrm{SNR}_{y 11,2}$ & 8.000 & 4.000 & 2.000 & 1.000 \\
\hline $\mathrm{SNR}_{y 12,2}$ & 12.500 & 6.250 & 3.125 & 1.562 \\
\hline
\end{tabular}


Table 6: Means and standard deviations $\chi^{2}$-test statistics for the example, $d f=130$ in all conditions.

\begin{tabular}{rrcc}
\hline$\theta_{i, i, g}$ & \multicolumn{1}{c}{$N$} & Mean & SD \\
\hline \multirow{4}{*}{0.500} & 200 & 172.163 & 20.677 \\
& 400 & 208.478 & 23.279 \\
& 800 & 246.368 & 26.614 \\
& 1000 & 284.059 & 29.115 \\
& 200 & 153.135 & 18.905 \\
& 400 & 170.194 & 20.104 \\
1.000 & 600 & 188.878 & 22.216 \\
& 800 & 207.508 & 23.731 \\
& 1000 & 226.344 & 25.513 \\
\hline & 200 & 143.301 & 17.874 \\
& 400 & 150.394 & 18.205 \\
2.000 & 600 & 159.114 & 19.431 \\
& 800 & 167.957 & 20.235 \\
& 1000 & 176.977 & 21.329 \\
\hline & 200 & 138.330 & 17.311 \\
& 400 & 140.411 & 17.174 \\
4.000 & 600 & 144.078 & 17.805 \\
& 800 & 148.052 & 18.167 \\
& 1000 & 152.101 & 18.797 \\
\hline
\end{tabular}




\section{Figures}
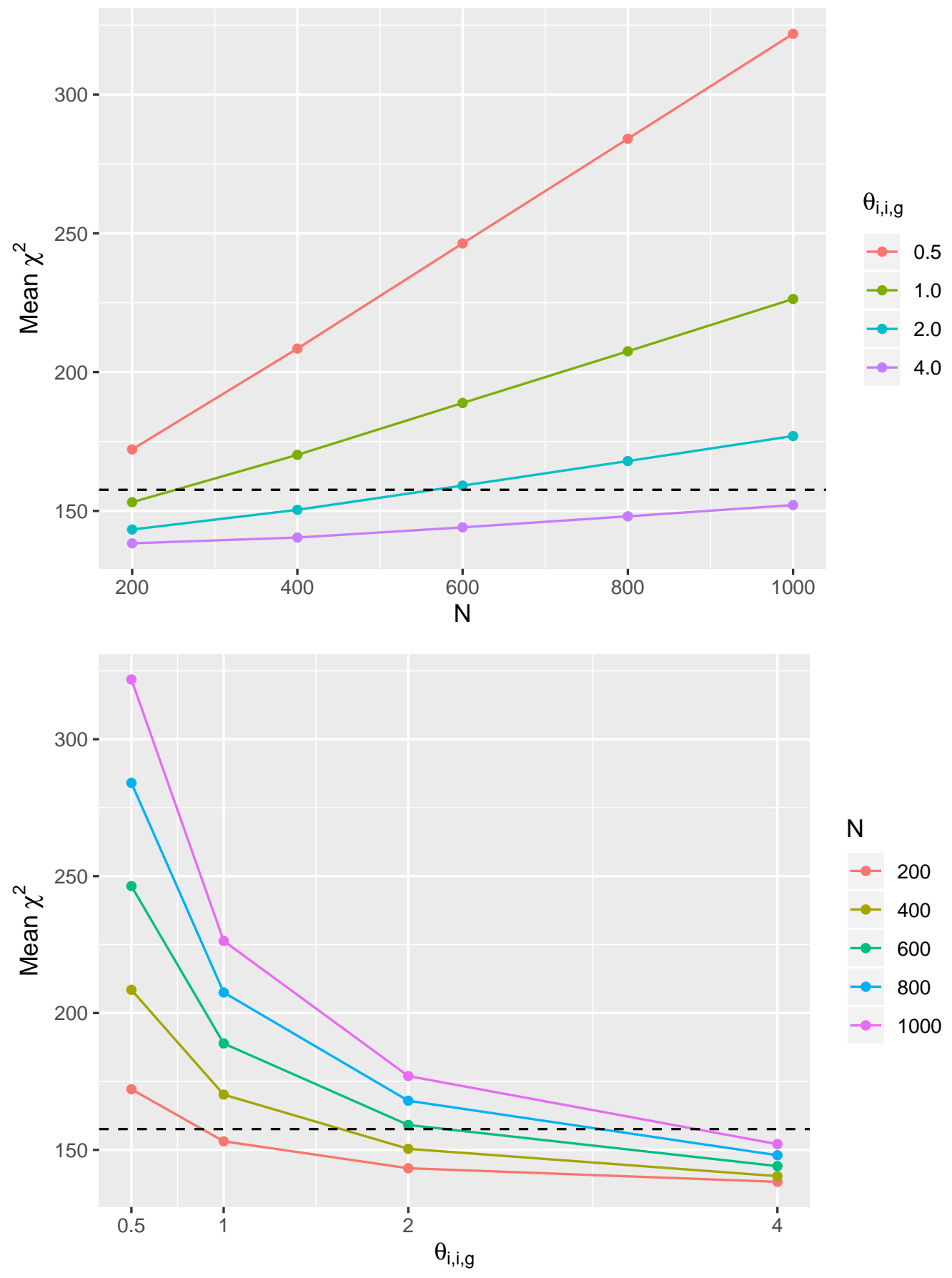

Figure 1: Results from the Monte Carlo study for the example in Raykov et al. (2020). Top panel: The mean $\chi^{2}$-test statistic is plotted against the sample size for the various $\theta_{i, i, g}$. Lower panel: The mean $\chi^{2}$-test statistic is plotted against $\theta_{i, i, g}$ for the various sample sizes. In both panels, the dashed line indicates the critical $\chi^{2}$-test statistic for $130 \mathrm{df}$. 